\title{
The topology of ice-sheet centres
}

\author{
J.F. NYE \\ H. H. Wills Physics Laboratory, University of Bristol, Bristol BS8 1TL, England
}

\begin{abstract}
An ice sheet will, in general, possess points where the horizontal component of velocity is zero, and some of these will be ice centres, occurring close to summits. The paper examines the possible flow patterns near such points. The corresponding horizontal strain-rate pattern is studied by considering an ice sheet which initially has perfect circular symmetry about a vertical axis. Before perturbation there is an isotropic point for the horizontal surface strain rate at the centre. It may be shown, on purely topological grounds and without any reference to the mechanism of flow, that, when the symmetry is broken, this point, being degenerate and structurally unstable, breaks up into two structurally stable components. The breakup always occurs in essentially the same way. Around the two component points the trajectories of principal strain-rate directions always have the lemon pattern. The contours of equal principal strain rate around them are usually hyperbolic; however, if the unperturbed flow pattern had a very pronounced spiral character, they would be elliptic. This behaviour is in contrast to that of the ice centre itself, which remains unsplit.
\end{abstract}

\section{INTRODUCTION}

The ideas of genericity and of structural stability, which originated as concepts within mathematics (Thom, 1975; Arnold, 1983, 1986; Guckenheimer and Holmes, 1983), have much to teach us in our attempts to model the natural world. In mathematics they have, of course, the rigour that is appropriate to that subject; in science it is natural that they should be applied in a somewhat looser way. In particular, they are of wide applicability in geophysics, where problems characteristically involve scalar, vector or tensor fields that have no special symmetry. An example in glaciology is the time-dependent behaviour of the edge of an irregular ice sheet (Nye, 1990). This paper studies the related problem of the behaviour of a flow centre in an irregular ice sheet.

The point of view to be adopted is unconventional in glaciology, and needs to be stated explicitly. The conventional way of treating ice sheets theoretically is first to set up a mathematical model, which is an idealization of the real ice sheet and which is chosen so as to be analytically or numerically tractable. The model will commonly contain assumptions about the flow law for ice, the isotropy of the ice, its thermal properties, the form and nature of the bed, the various boundary conditions, and so on. Here, however, we proceed differently.

Consider the surface of a real ice sheet at a particular time. A topographic map made at a certain scale represents a smoothed version of the real surface, and it is this smoothed surface that we deal with. Consider also the real two-dimensional horizontal velocity field of the ice at the ice-sheet surface, and let this now be smoothed at the same map scale. These smoothing operations completely define our model, and they represent the correspondence between model and reality. Thus, we are not dealing with an ice-sheet model in the usual sense, but with an ice sheet which is simply a smoothed version of a real one, such as the Antarctic or the Greenland ice sheet, in all its geographical complexity.

With such a general approach, it might be thought that little of value could be said, but this is not the case. Certain conclusions follow purely from topological considerations, which are absolute for generic smooth surfaces and functions. The word generic, as we use it in this paper, simply means typical. It refers to something that will occur when there are no special conditions. Likewise, non-generic means "of vanishingly small probability". This is admittedly a loose definition; in mathematics, as one would expect, generic has a strictly defined meaning, but such rigour would not be appropriate here.

The importance of the topological conclusions that follow from genericity derives precisely from the fact that they are independent of specific mechanisms of flow, and of specific models, in the usual sense of that term. Specific models, however good, are necessarily approximate; topological conclusions are absolute.

\section{STRUCTURAL STABILITY: MAXIMA, MINIMA AND SADDLES}

As an introductory example, let us consider how the surface of an ice sheet can change with time. The smoothed surface that we have constructed will generically show three kinds of points where the surface is level: maxima 


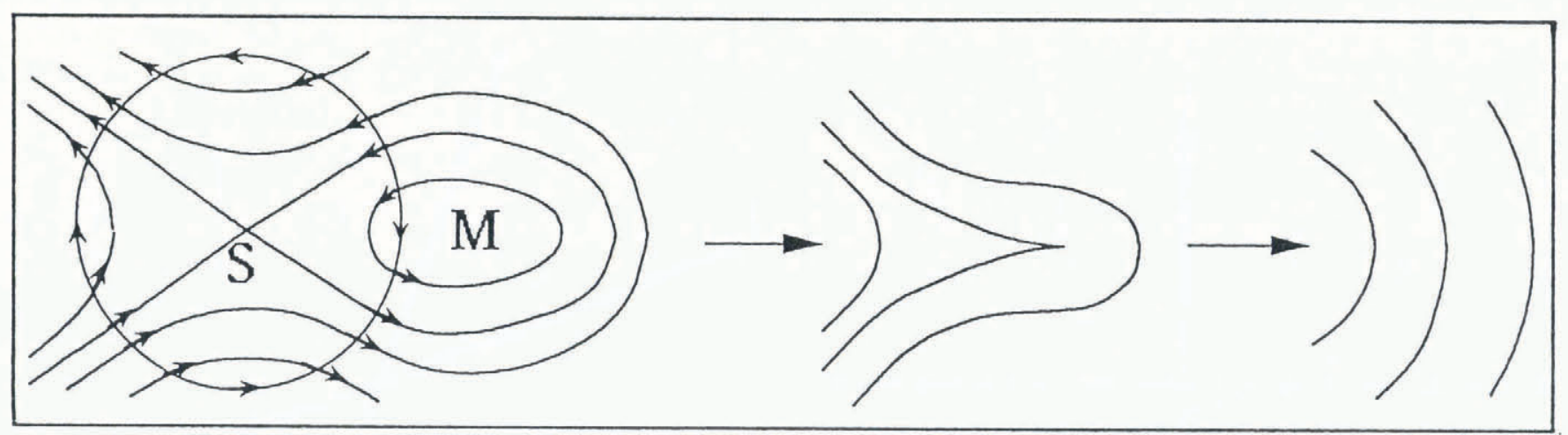

Fig. 1. Height contours of an ice sheet. A saddle $S$ (index -1 ) and a maximum $M($ index +1$)$ annihilate one another, the total singularity index remaining zero throughout.

(summits), saddles and minima (hollows), although the latter will occur rarely. At a slightly later time, as the ice sheet evolves, all these points will be found to have moved a little, but they will all retain their identities: they are said to be structurally stable. Generically, there are only two ways in which they can destroy themselves: (1) by moving to the edge of the ice sheet and disappearing, or (2) by a maximum or minimum colliding with a saddle to leave no level place (Fig. 1). If the lefthand diagram of Figure 1 represents a maximum and a saddle, the transition to the righthand diagram could be accomplished by progressively tilting the whole surface until eventually it all slopes downwards to the right.

\section{Singularity index}

It is helpful to view this last event by using the concept of the singularity index. To determine the index of a singularity such as the saddle $\mathrm{S}$ in Figure 1, draw a circuit around it (the circle) and note how the contour lines change direction as one proceeds once around the circuit. If the circuit is traversed clockwise, it will be seen that the contour direction (shown by the arrows) rotates by one revolution in the opposite sense; and this is so whatever the shape of the circuit, provided it encloses $\mathrm{S}$ and no other singularity. The singularity index is defined in this case as -1 . A corresponding clockwise circuit around $\mathrm{M}$ in Figure 1 (which may represent a maximum or a minimum) shows that the contour direction changes by one revolution clockwise: the index is accordingly defined as +1 . If, on the other hand, we travel clockwise on a circuit that encloses both S and $\mathrm{M}$, the contour direction shows no resultant rotation; the index is 0 . This illustrates the rule that the index for a circuit is the sum of the indices of the individual singularities that it encloses.

If we now keep a circuit fixed and make a small change in the contour pattern, the index for the circuit cannot change discontinuously (because its change results from adding many small continuous changes), unless a singularity should happen to pass across the circuit. Thus, provided this does not happen, the index for the circuit is conserved. For example, in Figure 1, S and M come together to leave no singularity; this is consistent with the result we get by noting the index of a circuit enclosing $\mathrm{S}$ and $\mathrm{M}$ (namely zero), and also noting the index of the same circuit after the annihilation event (namely zero again). It is clear from such considerations that, when singularities interact, their total index is conserved. This is the essential topological reason why maxima and minima on an ice sheet can only be destroyed by collision with saddles (or by passing over the boundary).

\section{DIRECTIONS OF ICE FLOW}

Consider now, at a given instant, the smoothed twodimensional distribution of horizontal velocity at the surface of the ice sheet, represented by the two-dimensional vector field $\mathbf{u}(\mathbf{r})$, where $\mathbf{r}=(x, y)$ denotes position. As in any such field there are, in general, points where $\mathbf{u}(\mathbf{r})=0$. An ice centre is a point where $\mathbf{u}(\mathbf{r})=0$ and where the two principal horizontal strain rates $e_{1}$ and $e_{2}$ are both positive. (It is non-generic for either of them to be zero precisely at a point where $\mathbf{u}(\mathbf{r})$ is also zero. That would be a structurally unstable situation; for example, a change in the scale of smoothing would destroy the coincidence.)

Although an ice centre will normally occur near a summit, it is non-generic that it should coincide with a summit exactly; this would entail conditions so special that they would not be found in Nature. Similarly, it is non-generic that the rotation rate at an ice centre should be exactly zero, although it may very well be quite small. Most detailed ice-sheet models are based on a flow rule of the type

$$
\mathbf{u}(\mathbf{r})=s(\mathbf{r}) \operatorname{grad} \phi(\mathbf{r})
$$

where $\phi(\mathbf{r})$ is the height of the surface, and $s(\mathbf{r})$ is some scalar function of position (which would include, for example, a dependence on bed roughness, as well as on powers of $|\operatorname{grad} \phi(r)|$ and of ice thickness). This is the most general rule that ensures that $\mathbf{u}(\mathbf{r})$ is perpendicular to the surface contours (Nye, 1952; Hutter, 1983, p. 459 ), as is commonly observed to be approximately the case. In such a model, an ice centre would necessarily occur exactly at a summit $(\operatorname{grad} \phi=0)$. It follows from rule (1) that, since curl grad $=0$,

$$
\operatorname{curl} \mathbf{u}=\operatorname{grad} s(\mathbf{r}) \times \operatorname{grad} \phi(\mathbf{r}) .
$$

Thus, with this rule, not only would there be no velocity at the summit, but no rotation either. However, there would be rotation wherever $\operatorname{grad} s(\mathbf{r})$ is not parallel or anti-parallel to $\operatorname{grad} \phi(\mathbf{r})$. It is useful to note these consequences of rule (1) but, since the rule is only approximate and our aim is to avoid all approximation, we shall not, in fact, apply any such constraint in what follows. 

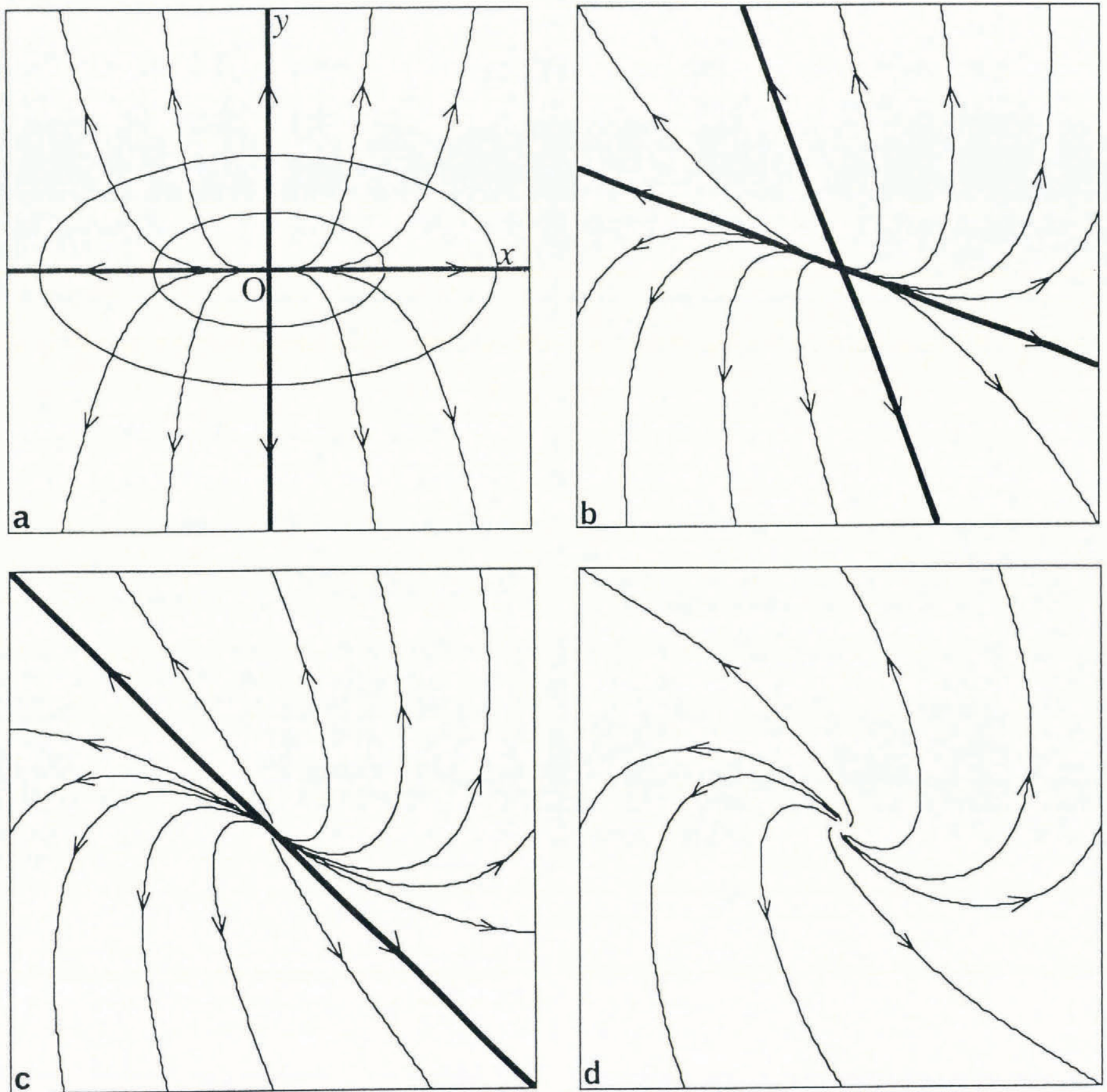

Fig. 2. Flowline'patterns at an ice centre for varying amounts of rotation. The ratio of the principal strains $e_{2} / e_{1}$ is taken as 4 . The straight flowlines come together as $\omega$ increases. (a) $\omega=0$, (b) $\omega=e_{1}$, (c) $\omega=1.5 e_{1}$, the critical value, (d) $\omega=2.22 e_{1}$.

Our point of view is that there is nothing to prevent a rotation at an ice centre, and therefore it will occur in Nature, even if only to a small degree. A possible physical mechanism is provided by the nature of the ice-sheet bed: this will necessarily favour either clockwise or anticlockwise motion; it will never favour both equally, for that would be non-generic. Of course, this is only one of a large number of possible physical mechanisms.

Taking an ice centre as origin $\mathrm{O}$ and $x, y$ axes parallel to the principal horizontal strain rates $e_{1}$ and $e_{2}$ at $\mathrm{O}$, the velocity components $u(x, y)$ and $v(x, y)$, at a given instant, are given to lowest order by

$$
\left.\begin{array}{l}
u=e_{1} x-\omega y \\
v=\omega x+e_{2} y
\end{array}\right\}
$$

where $\omega$ is the instantaneous rate of rotation at O. To find the possible patterns of instantaneous flowlines, we write $u=\dot{x}, v=\dot{y}$, thus

$$
\left.\begin{array}{l}
\dot{x}=e_{1} x-\omega y \\
\dot{y}=\omega x+e_{2} y
\end{array}\right\} .
$$

If the velocity distribution were steady with time, these equations would give the actual particle motions. However, generically, the velocity distribution changes with time, and so the equations merely describe the instantaneous motion of the particles. We now follow a standard procedure from dynamical systems analysis (Pippard, 1985, p. 22). First eliminate $y$ to give

$$
\ddot{x}-T \dot{x}+\Delta x=0
$$


where $T$ is the trace, $e_{1}+e_{2}$, and $\Delta$ the determinant, $e_{1} e_{2}+\omega^{2}$, of the matrix of coefficients in Equations (2). Since Equation (3) is the equation for damped harmonic motion, and $T$ is positive, the solution grows exponentially:

if $\Delta<\frac{1}{4} T^{2}$, i.e. $4 \omega^{2}<\left(e_{1}-e_{2}\right)^{2}$, the solution grows in a non-oscillatory way (nodal instability);

if $\Delta>\frac{1}{4} T^{2}$, i.e. $4 \omega^{2}>\left(e_{1}-e_{2}\right)^{2}$, it grows in an oscillatory way (focal instability).

The resulting flowline patterns are drawn in Figure $2 \mathrm{a}, \mathrm{b}, \mathrm{c}$ and $\mathrm{d}$ for $e_{2} / e_{1}=4$. When $|\omega|$ is below the critical value given by $4 \omega^{2}=\left(e_{1}-e_{2}\right)^{2}$, there are two straight flowlines, generically not at right-angles, one of them being a common tangent at $\mathrm{O}$ to all the other flowlines. When $|\omega|$ is above the critical value, the flowlines cease to possess a common tangent. We expect the rotation rate to be small at an ice centre, as already discussed, and therefore a pattern between those of Figure 2a and $\mathrm{b}$ is to be expected. As time progresses and the ice sheet evolves, an ice centre will move, but because it is structurally stable the general features of its local flowline pattern will be preserved.

If rule (1) were obeyed, the ice centre would be exactly at a summit and there would be no instantaneous rotation at this point. It is readily shown that in this case the contour lines for the surface would be concentric ellipses of the same shape as the representation quadric for strain rate, namely

$$
e_{1} x^{2}+e_{2} y^{2}=1
$$

These are the ellipses shown in Figure 2a. The instantaneous flowlines would be the one-parameter $(A)$ set of curves

$$
y=A x^{m} \quad \text { where } m=e_{2} / e_{1}
$$

which are drawn in Figure 2a for $e_{2} / e_{1}=4$. They would fan out from the direction of lesser principal strain rate.

A similar treatment can be given for the flowlines around a zero-velocity point with $e_{1}$ and $e_{2}$ having opposite signs, as would be expected near a surface saddle. If rule (1) applied, $\mathbf{u}$ would be exactly zero at a surface saddle, and so would $\omega$. Therefore, we expect a $\mathbf{u}=0$ point near a surface saddle and that $\omega$ will be small at this point. Since $|\omega|$ is likely to be small compared with $\left|e_{1}\right|$ and $\left|e_{2}\right|$, we expect the determinant $\Delta$ to be negative, and therefore, in dynamical systems terminologv, a saddle-point instability. However, if, exceptionally, $|\omega|$ were large enough $\left(\omega^{2}>-e_{1} e_{2}\right)$, four other possibilities would appear as follows (see Pippard, 1985, p. 22 for terminology):

$$
\begin{aligned}
& e_{1}+e_{2}>0, \quad \omega^{2}>\frac{1}{4}\left(e_{1}-e_{2}\right)^{2}, \\
& e_{1}+e_{2}>0, \quad-e_{1} e_{2}<\omega^{2}<\frac{1}{4}\left(e_{1}-e_{2}\right)^{2}, \quad \mathrm{ni} \\
& e_{1}+e_{2}<0, \quad-e_{1} e_{2}<\omega^{2}<\frac{1}{4}\left(e_{1}-e_{2}\right)^{2}, \quad n s \\
& e_{1}+e_{2}<0, \quad \omega^{2}>\frac{1}{4}\left(e_{1}-e_{2}\right)^{2}, \quad \text { fs }
\end{aligned}
$$
ns

where "fi" means focal instability, "ni" means nodal instability, "ns" means nodal stability and "fs" means focal stability.

\section{PATTERNS OF STRAIN RATE}

The pattern of horizontal strain-rate directions near an ice centre behaves quite differently from the pattern of flowlines. To explain this, it is convenient to begin with an ice sheet that is circularly symmetric in plan view in all its features (topography, accumulation rate, temperature, etc.). Of course, this is quite non-generic, but we shall in a moment perturb the ice sheet in a general way. Before perturbation, the ice spreads out radially from the centre in all directions and the horizontal strain rate $e_{i j}(i, j=x, y)$ at the centre is isotropic:

$$
\mathbf{e}=\left(e_{i j}\right)=\left(\begin{array}{cc}
K & 0 \\
0 & K
\end{array}\right),
$$

say, the constant $K$ being positive. (At an isotropic point

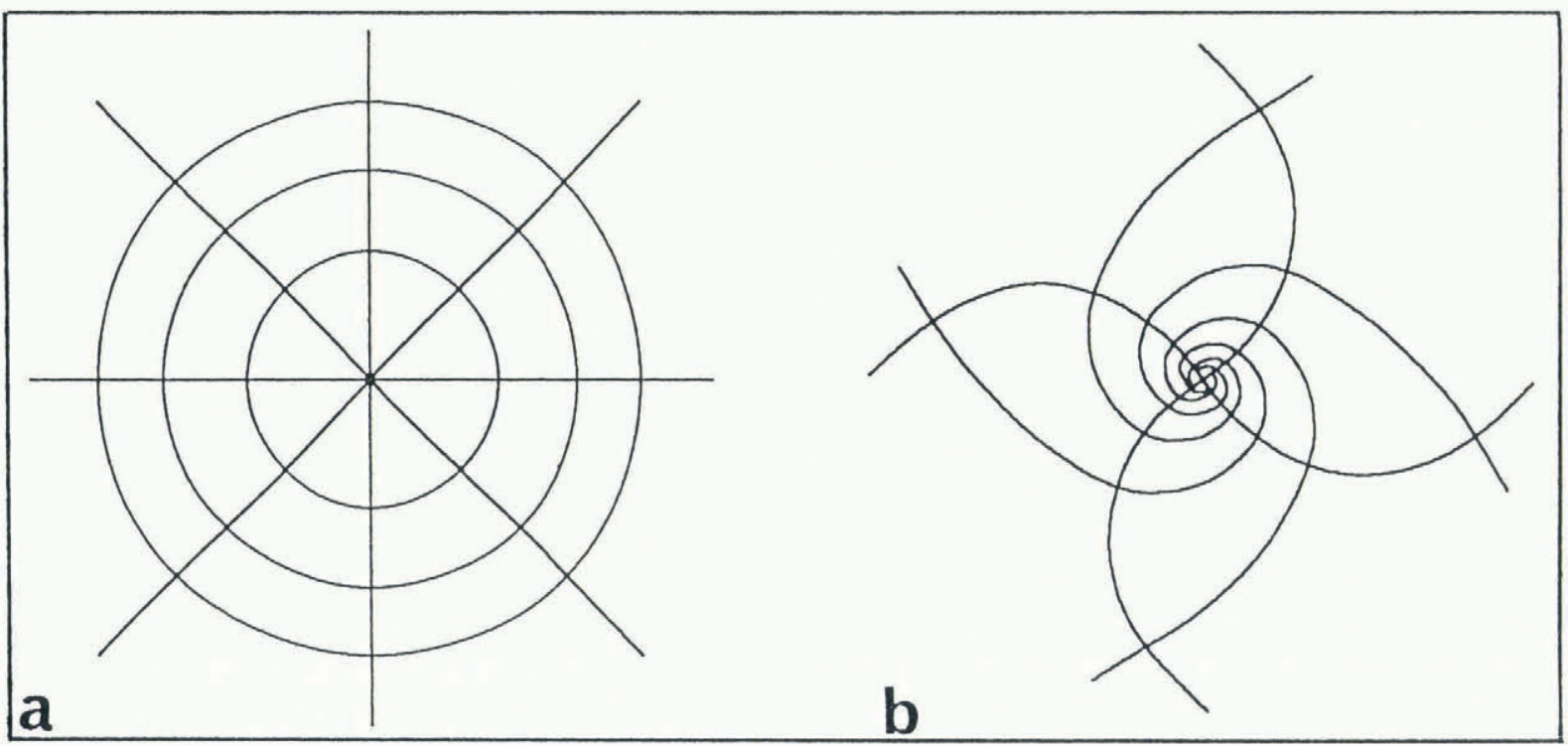

Fig. 3. Trajectories of principal strain rate for a circularly symmetric ice sheet, (a) without, and

(b) with rotation. 


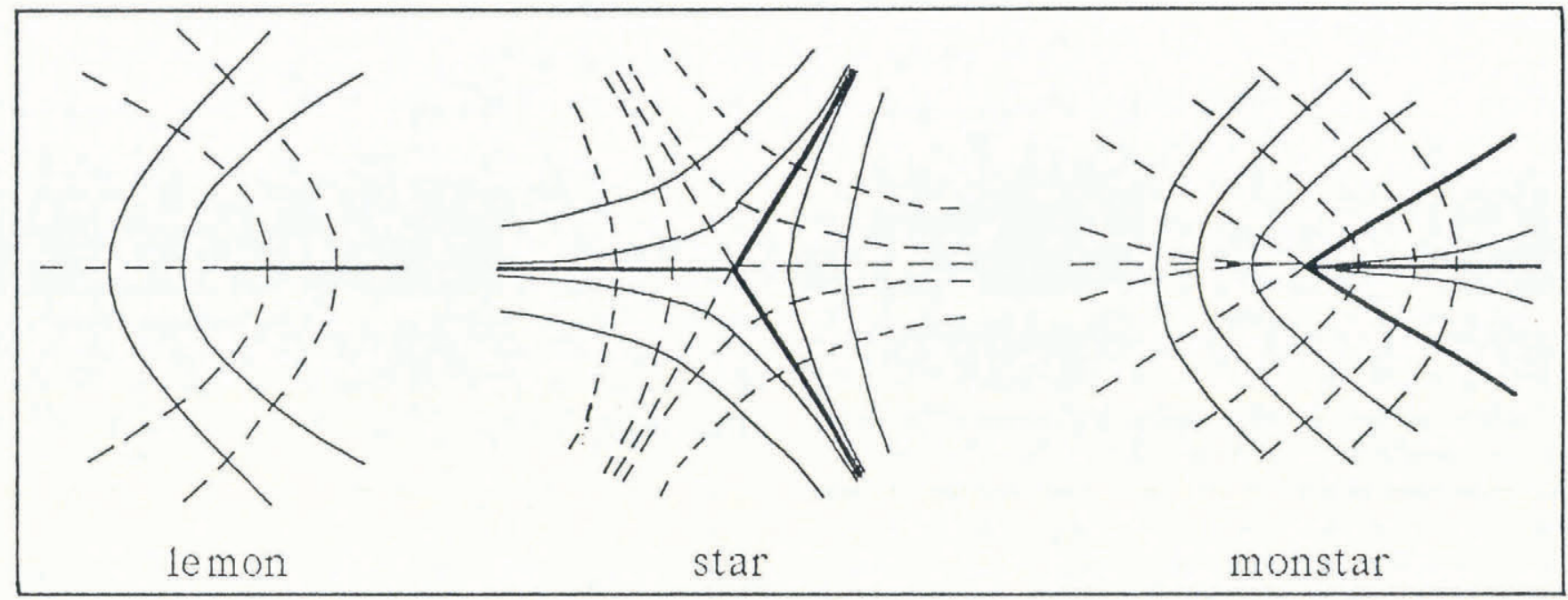

Fig. 4. The pattern of principal strain-rate trajectories around an isotropic point can, in general, be of three different kinds. The lemon and monstar patterns have index $+\frac{1}{2}$, while the star pattern has index $-\frac{1}{2}$. On the other hand, the star and monstar have three straight trajectories, while the lemon has only one. The nomenclature is due to Berry and Hannay (1977).

for strain rate, in general, e has this form, but $K$ need not be positive.)

Away from the centre the strain will not be isotropic, and in the simplest case the trajectories of principal strain rate would be radial lines and circles (Fig. 3a). However, circular symmetry would still be retained if the trajectories had a spiral character, as in Figure 3b. It can be shown that this implies that the flowlines are spirals also. In addition, there is a rotation rate that varies with radial distance. (These effects involve terms two orders higher than were considered in section 3 , that is, cubic terms in Equations (2).)

The singularity index of the patterns of orthogonal strain-rate directions in Figure $3 \mathrm{a}$ and $\mathrm{b}$ is +1 . However, the singularity index for the trajectory pattern of an isotropic point, in general, is $\pm \frac{1}{2}$, as can be seen by inspection of the patterns in Figure 4 (Nye, 1983, 1986a). (The reason for the $\frac{1}{2}$, as compared with 1 for the contour patterns, is that rotation of the strain-rate tensor at a point by $\pi$ brings it into self-coincidence, whereas a contour, which has a high side and a low side, has to be rotated by $2 \pi$ to be brought into self-coincidence.) Therefore, we can expect that, on perturbation, our +1 isotropic point will split into two, each of index $+\frac{1}{2}$. In other words, the +1 isotropic point is structurally unstable. (A similar splitting occurs with the isotropic points for curvature situated at each end of the axis of revolution of a spheroid; when the rotational symmetry is broken, each of these splits into two. In crystal optics the transition from a uniaxial crystal to a biaxial crystal is somewhat similar. However, these analogies are not perfect, as we shall see.) After perturbation, the local flowline pattern will be like those of Figure 2; unlike the central isotropic point, the flow centre is not split.

In principle, the two isotropic points for strain rate could be lemon or monstar (Fig. 4), but not star, because this has index $-\frac{1}{2}$. Isotropic points are also characterized by the contour property. Around each isotropic point one can draw contours of equal principal strain rate (lines along which a principal strain rate $e_{1}$ or $e_{2}$ takes a fixed value). There are two possibilities: the contours for both $e_{1}$ and $e_{2}$ are either two families of ellipses or two families of hyperbolas. Thus, in principle, not only could our two isotropic points be lemon or monstar, but in addition they could be elliptic or hyperbolic. We now show that they are, in fact, always lemon and nearly always hyperbolic.

To represent the unperturbed $e_{i j}$ within a small neighbourhood of the central degenerate isotropic point, taken as the origin, write the quadratic expansion

$$
\mathbf{e}=\left(\begin{array}{cc}
K+a x^{2}+b y^{2}+c x y & d x^{2}+p y^{2}+f x y \\
d x^{2}+p y^{2}+f x y & K+g x^{2}+h y^{2}+k x y
\end{array}\right)
$$

(the strain-rate distribution is considered smooth for the same reason as the surface topography). Linear terms have been omitted, because the pattern is centrosymmetric and therefore the tensor must be invariant under the transformation $(x, y) \rightarrow(-x,-y)$. Circular symmetry of the kind shown in Figure 3a requires, further, that (a) the directions of principal strain rate are radial and circumferential, and (b) the two strain-rate invariants are functions of $r$ and not $\theta(r, \theta$ being polar coordinates). A short analysis shows that these conditions reduce $\mathbf{e}$ to the form

$$
\mathbf{e}=\left(\begin{array}{cc}
K+a x^{2}+b y^{2} & (a-b) x y \\
(a-b) x y & K+b x^{2}+a y^{2}
\end{array}\right) \quad(a \neq b) .
$$

If, however, we admit a rotation, as in the generic case of Figure $3 \mathrm{~b}$, there are additional terms involving $\mathrm{c}$ :

$$
\mathbf{e}=\left(\begin{array}{ll}
p_{0} & r_{0} \\
r_{0} & q_{0}
\end{array}\right)
$$

where

$$
\begin{aligned}
& p_{0}=K+a x^{2}+b y^{2}+c x y \\
& q_{0}=K+b x^{2}+a y^{2}-c x y \\
& r_{0}=-\frac{1}{2} c\left(x^{2}-y^{2}\right)+(a-b) x y .
\end{aligned}
$$


Working with this more general form, we now perturb it by a small amount in lowest order to make the origin non-isotropic, thus:

$$
\mathbf{e}=\left(\begin{array}{ll}
p_{1} & r_{1} \\
r_{1} & q_{1}
\end{array}\right)
$$

where

$$
\begin{aligned}
& p_{1}=K+\epsilon_{1}+a x^{2}+b y^{2}+c x y \\
& q_{1}=K-\epsilon_{1}+b x^{2}+a y^{2}-c x y \\
& r_{1}=\epsilon_{2}-\frac{1}{2} c\left(x^{2}-y^{2}\right)+(a-b) x y
\end{aligned}
$$

where $\epsilon_{1}$ and $\epsilon_{2}$ are small constants. "Small" means that the separation of the new isotropic points is small compared with a characteristic radial dimension of the original strain-rate distribution. (A more general perturbation in $q_{1}$, by $\epsilon_{3}$, say, rather than by $-\epsilon_{1}$, would have no effect other than to change the mean normal strain rate at the origin.) It is necessary to impose one further condition: because the strain-rate tensor is the gradient of a single-valued velocity function, its components must satisfy what in elasticity would be called the equations of compatibility. In two dimensions, as here, they reduce to the single condition:

$$
\frac{\partial^{2} e_{x x}}{\partial y^{2}}+\frac{\partial^{2} e_{y y}}{\partial x^{2}}=2 \frac{\partial^{2} e_{x y}}{\partial x \partial y} .
$$

Applied to Equation (7) this gives $a=3 b$, so that the tensor now has the form

$$
\mathbf{e}=\left(\begin{array}{ll}
p_{2} & q_{2} \\
q_{2} & r_{2}
\end{array}\right)
$$

where

$$
\begin{aligned}
& p_{2}=K+\epsilon_{1}+b\left(3 x^{2}+y^{2}\right)+c x y \\
& q_{2}=K-\epsilon_{1}+b\left(x^{2}+3 y^{2}\right)-c x y \\
& r_{2}=\epsilon_{2}-\frac{1}{2} c\left(x^{2}-y^{2}\right)+2 b x y .
\end{aligned}
$$

The conditions for an isotropic point, $e_{x x}=e_{y y}$, $e_{x y}=0$, reveal that there are now two such points symmetrically placed and, without loss of generality, we can make them occur on the $x, y$ axes (different from those of section 3 because $\epsilon_{2} \neq 0$ ) by setting

$$
\epsilon_{2}=-\frac{c}{2 b} \epsilon_{1} .
$$

Taking a suitable sign for $\epsilon_{1}$, they now occur at $x=$ $\pm\left(-\epsilon_{1} / b\right)^{\frac{1}{2}}= \pm \epsilon$ say, and $y=0$. Shifting the origin to $x=+\epsilon, y=0$, and retaining only the linear terms in the expansion about this point, we obtain

$$
\mathbf{e}=\left(\begin{array}{cc}
K^{\prime}+6 \epsilon b X+\epsilon c Y & -\epsilon c X+2 \epsilon b Y \\
-\epsilon c X+2 \epsilon b Y & K^{\prime}+2 \epsilon b X-\epsilon c Y
\end{array}\right)
$$

where $K^{\prime}$ is a constant, $X=x-\epsilon, Y=y$. To characterize the isotropic point, we use the discriminants given in the Appendix. Thus, to check the singularity index, we evaluate the discriminant $D_{\mathrm{I}}$ to find

$$
D_{\mathrm{I}}=8 \epsilon^{2} b^{2}+2 \epsilon^{2} c^{2} .
$$

Since this is always positive, the index is $+\frac{1}{2}$, as was anticipated.

To decide whether there are one or three straight trajectories passing through the point (straight meaning zero curvature at the point itself), that is, whether the pattern is lemon or monstar, we evaluate the line discriminant $D_{\mathrm{L}}$ (see Appendix). Thus

$$
\operatorname{sgn} D_{\mathrm{L}}=-\operatorname{sgn}\left(4 b^{2}+c^{2}\right) .
$$

Since $D_{\mathrm{L}}$ is always negative, the line number is 1 and the pattern is lemon rather than monstar.

For the contour discriminant $D_{\mathrm{C}}$ (see Appendix) that decides whether the contours of constant principal strain rate are elliptic or hyperbolic, we find

$$
\operatorname{sgn} D_{\mathrm{C}}=-\operatorname{sgn}\left(12 b^{2}-c^{2}\right) .
$$

Thus, if $c^{2}<12 b^{2}$, the contours are hyperbolic and, if $c^{2}>12 b^{2}$, they are elliptic. Recalling that $c$ is the parameter that controls whether the pattern is spiral or not, we see that, unless the spiral quality is pronounced, the contours are hyperbolic; the elliptic case would be rare. It may be surprising that the contours at the isotropic points can be hyperbolas when the unperturbed contours were simply concentric circles; the details of the transition are illustrated in Nye (1991).

Although the tensor in Equation (9) with condition (10) contains four parameters, namely $K, \epsilon_{1}, b, c$, the pattern of principal strain-rate directions it describes is, in fact, entirely controlled by a single parameter, apart from an overall scaling. To see this, first subtract off the isotropic strain rate $K$ and divide the remaining strain rate by $\epsilon_{1}$, neither of which operations affects the pattern. Then apply the isotropic scaling

$$
x=\left(-\frac{\epsilon_{1}}{b}\right)^{\frac{1}{2}} X, \quad y=\left(-\frac{\epsilon_{1}}{b}\right)^{\frac{1}{2}} Y,
$$

to give, in place of Equation (9), the strain-rate tensor

$$
\left(\begin{array}{cc}
1-3 X^{2}-Y^{2}-2 c^{\prime} X Y & -c^{\prime}\left(1-X^{2}+Y^{2}\right)-2 X Y \\
-c^{\prime}\left(1-X^{2}+Y^{2}\right)-2 X Y & -1-X^{2}-3 Y^{2}+2 c^{\prime} X Y
\end{array}\right)
$$

where $c^{\prime}=c / 2 b$. In this form the only free parameter is $c^{\prime}$, and the isotropic points occur at $X= \pm 1, Y=0$. Figure $5 \mathrm{a}, \mathrm{b}, \mathrm{c}$ and $\mathrm{d}$ shows the patterns of principal strain-rate directions for $c^{\prime}=0,0.5,1,10$. Notice that it is only for $c^{\prime}=0$ (i.e. $c=0$ ) that the two lemons share a common trajectory. Such sharing would not be typical in Nature. Generically, the lines from the two points miss one another, and the whole pattern has a spiral character, however slight this may be in practice. The change-over from hyperbolic to elliptic takes place at $c^{\prime}=\sqrt{3}$.

We have not been specific about the nature of the small perturbation that acted on the original degenerate isotropic point at the centre of a circular ice sheet; this is precisely because it does not matter, so long as the perturbation is such as to break the original circular symmetry, leaving either none at all, or, at most, two-fold symmetry (an example of what happens when four-fold symmetry is preserved by the perturbation is shown in 

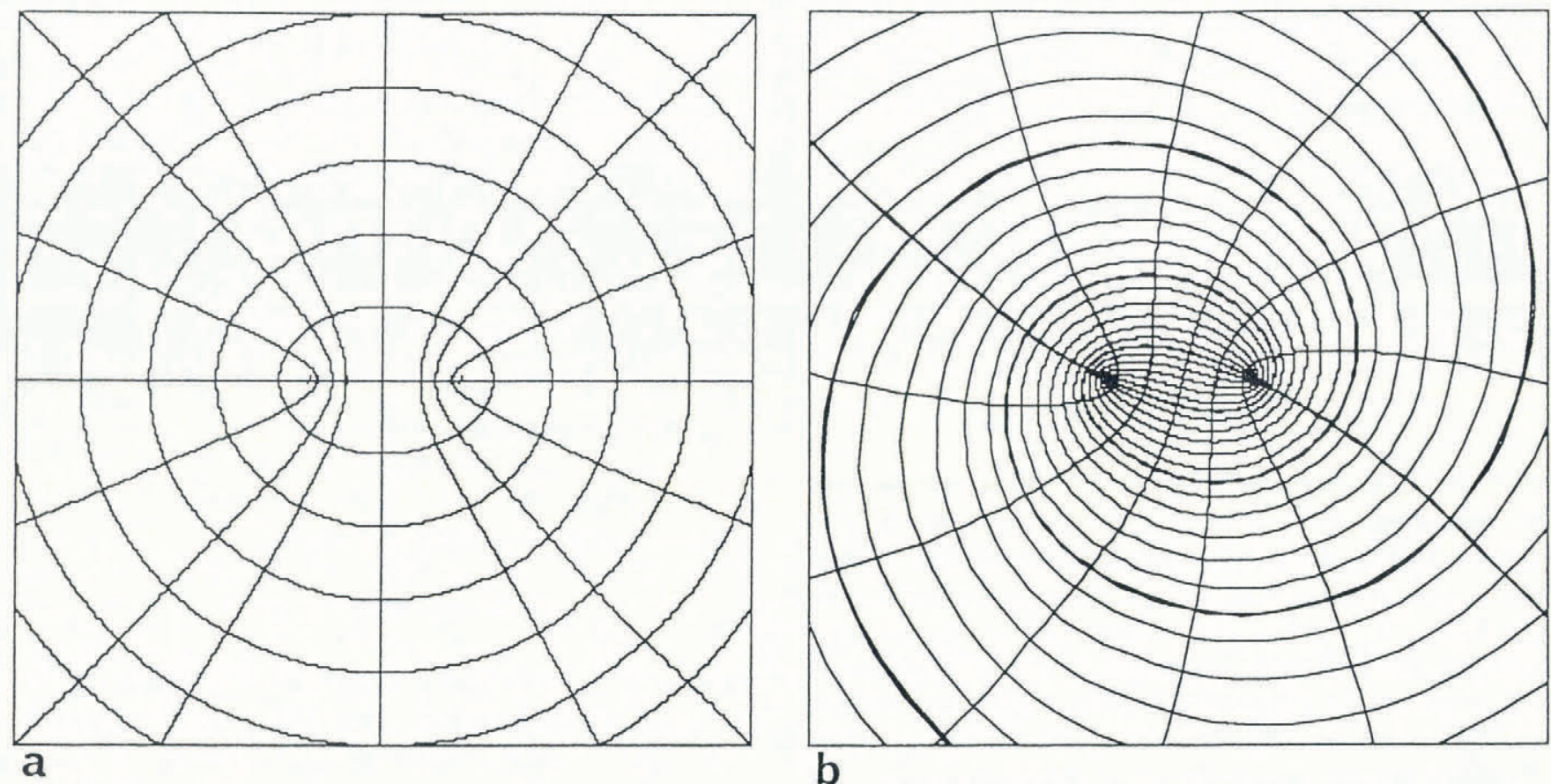

b
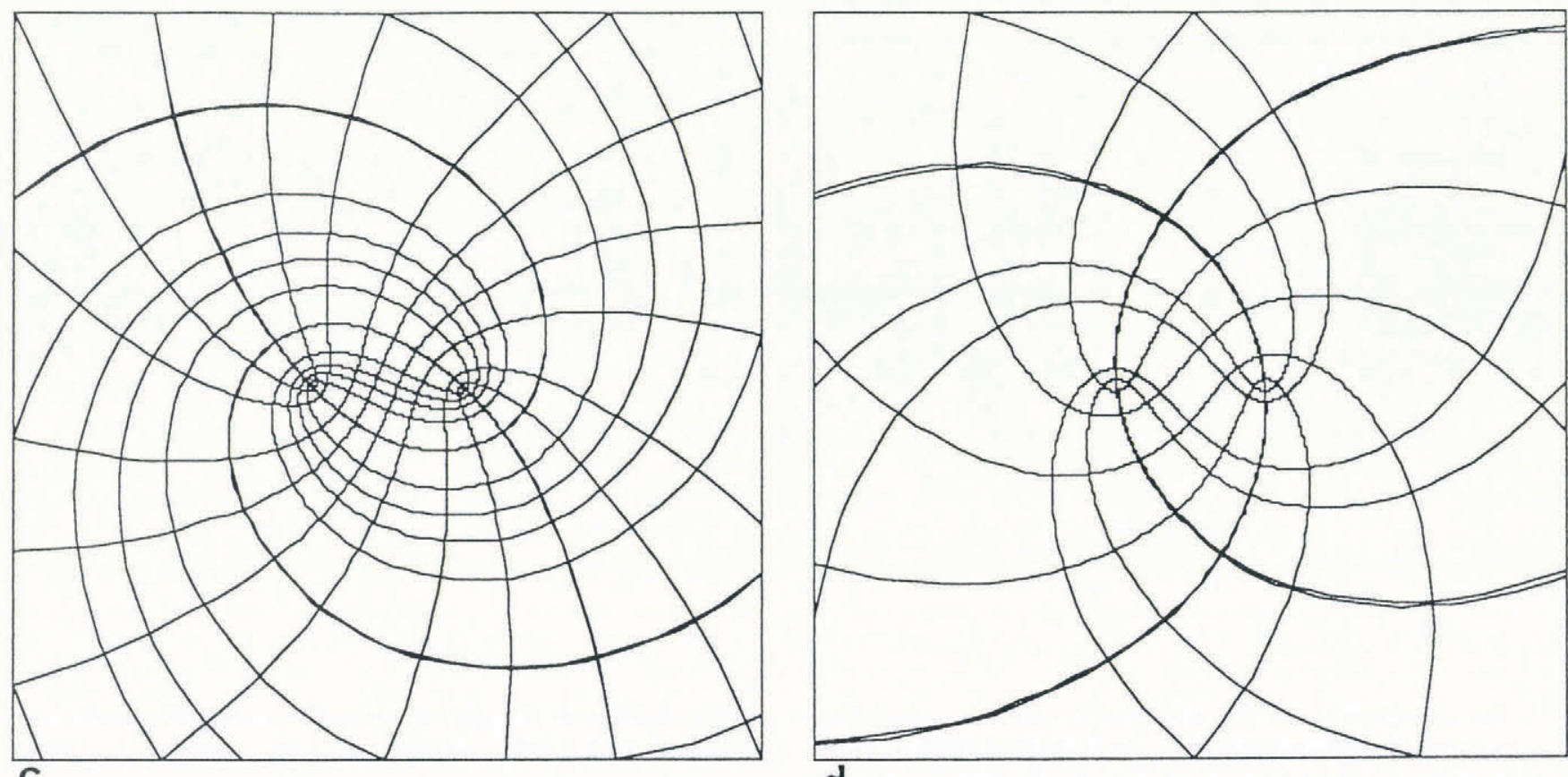

Fig. 5. Patterns of principal strain-rate directions (Fig. 3b) after perturbation. The values of $c^{\prime}$ are (a) 0 , (b) 0.5 , (c) 1 , (d) 10. The trajectories were integrated by a low-order Runge-Kutta method with an interval proportional to the difference of the two principal values.

figure 13 of Nye (1986a)). The parameter $\epsilon$, which is called a control parameter, could represent time, so that we should then be watching the evolution of the ice sheet. More interestingly, it could represent the scale of resolution on which we choose to observe, that is, the map scale. Viewed from a distance, the patterns in Figure $5 \mathrm{a}, \mathrm{b}, \mathrm{c}$ and $\mathrm{d}$ are those of the unperturbed strain rate. Thus, generically, a pattern which appears, on a certain map scale, to be as in Figure 3b will inevitably be found, on higher resolution, to be broken up in the way shown by Figure $5 \mathrm{~b}, \mathrm{c}$ and $\mathrm{d}$. (Likewise, a pattern which, on a certain scale, shows no isotropic points may be found, as the resolution is increased, to contain one or more close pairs of isctropic points of opposite index.) As another example of the interpretation of $\epsilon$, suppose we are considering a circularly symmetric ice sheet modelled numerically, and suppose $\epsilon$ is some constant which breaks the symmetry of the model when it is changed (it might be a constant governing the temperature distribution, for example). Then the central degenerate isotropic point will break up in the way we have calculated.

\section{CONCLUSIONS}

We have shown, on purely topological grounds without reference to the mechanism of flow, that when the perfect 
symmetry of a circular ice sheet is perturbed in a general way the isotropic point for strain rate at its centre, being structurally unstable, breaks up into two structurally stable components. Around them, the strain-rate trajectories always have the lemon pattern, and the contours of equal principal strain rate are usually hyperbolic. This splitting is in contrast to the behaviour of the ice centre itself, which remains single and intact.

As the ice sheet becomes progressively less circular, we expect the two isotropic points to separate further, and, since their indices cannot change discontinuously, they will remain $+\frac{1}{2}$. Meanwhile, new isotropic points will be born together as twins of opposite index, or will enter the ice sheet at its boundary. Thus, the original isotropic points will continue to exist until either they interact with one of the new ones or escape across the boundary. Although any one isotropic point retains its index (between interactions), nevertheless its line and contour character can change.

Of course, the distribution of the horizontal component of velocity in an ice sheet is only one example of a two-dimensional flow field which exhibits phenomena similar to those described here; many other examples could be envisaged. We also remark, without proof (to be published), that there is a perfect analogy between the breakup of the central isotropic point and the behaviour of the focus of a slightly imperfect lens used with circularly polarized light. The strain-rate ellipse is analogous to the polarization ellipse. However, the analogy with the breakup of a circularly symmetric degenerate umbilic point of a surface is not exact because of the presence of the parameter $c$.

\section{REFERENCES}

Arnold, V. I. 1983. Geometrical methods in the theory of ordinary differential equations. Berlin, SpringerVerlag, chapter 12.

Arnold, V.I. 1986. Catastrophe theory. Second edition. Berlin, Springer-Verlag, chapter 12.

Berry, M. V. and J.H. Hannay. 1977. Umbilic points on Gaussian random surfaces. J. Phys. A, 10(11), 18091821.

Guckenheimer, J. and P. Holmes. 1983. Non-linear oscillations, dynamical systems, and bifurcations of vector fields. New York, Springer-Verlag.

Hutter, K. 1983. Theoretical glaciology; material science of $i c e$ and the mechanics of glaciers and ice sheets. Dordrecht, etc., D. Reidel Publishing Company/Tokyo, Terra Scientific Publishing Company.

Nye, J.F. 1952. A method of calculating the thicknesses of the ice-sheets. Nature, 169(4300), 529-530.

Nye, J.F. 1983. Monstars on glaciers. J. Glaciol., 29(101), 70-77.

Nye, J. F. 1986a. Isotropic points on glaciers. J. Glaciol., 32(112), 363-365.
Nye, J.F. 1986b. The catastrophe optics of liquid drop lenses. Proc. R. Soc. London, Ser. A, 403,1-26.

Nye, J.F. 1990. Interpreting the field evidence of past ice sheets: structural stability and genericity. Ann. Glaciol., 14, 208-210.

Nye, J.F. In press. A topological approach to the strainrate pattern of ice sheets. J. Glaciol. (Grenoble meeting, 23 November 1990, in honour of Professor L. Lliboutry.)

Pippard, A.B. 1985. Response and stability. Cambridge, Cambridge University Press.

Thom, R. 1975. Structural stability and morphogenesis. Reading, MA, Benjamin.

Thorndike, A. S., C. R. Cooley and J. F. Nye. 1978. The structure and evolution of flow fields and other vector fields. J. Phys. A, 11, 1455-1490.

The accuracy of references in the text and in this list is the reponsibility of the author, to whom queries should be addressed.

\section{APPENDIX}

The field of any symmetric tensor in the neighbourhood of an isotropic point can be written up to linear terms as

$$
\left(\begin{array}{cc}
K+\alpha x+\beta y & \beta_{1} x+\gamma_{1} y \\
\beta_{1} x+\gamma_{1} y & K+\gamma x+\delta y
\end{array}\right) .
$$

The isotropic point is classified by the following discriminants (Thorndike and others, 1978, p. 1489):

Singularity index:

$$
D_{\mathrm{I}}=(\alpha-\gamma) \gamma_{1}+(\delta-\beta) \beta_{1}>0 \quad+\frac{1}{2} \text { (lemon or monstar), }
$$

Contours of constant principal values:

$$
\begin{aligned}
D_{\mathrm{C}}=4\left(\alpha \gamma-\beta_{1}^{2}\right)\left(\beta \delta-\gamma_{1}^{2}\right) & \\
-\left(\alpha \delta+\beta \gamma-2 \beta_{1} \gamma_{1}\right)^{2}>0 & \text { elliptic, } \\
<0 & \text { hyperbolic } .
\end{aligned}
$$

Line number:

$$
\begin{aligned}
D_{\mathrm{L}}= & 4\left[3 \gamma_{1}\left(\alpha-\gamma-\gamma_{1}\right)-\left(\delta-\beta-\beta_{1}\right)^{2}\right] \\
& \times\left[3 \beta_{1}\left(\delta-\beta-\beta_{1}\right)-\left(\alpha-\gamma-\gamma_{1}\right)^{2}\right] \\
& -\left[\left(\delta-\beta-\beta_{1}\right)\left(\alpha-\gamma-\gamma_{1}\right)-9 \beta_{1} \gamma_{1}\right]^{2} \\
>0 & 3 \text { (star or monstar }), \\
& <0 \quad 1 \text { (lemon). }
\end{aligned}
$$

If, in addition, the components are the second derivatives (Hessian) of a potential, so that $\beta=\beta_{1}$ and $\gamma=\gamma_{1}$ (as is the case, for example, for the curvature tensor of a surface), the above discriminants reduce to those given by Berry and Hannay (1977). 